\title{
THE POINT DISCHARGE IN AIR FOR PRESSURES GREATER THAN ATMOSPHERIC.
}

By O. Amsden Gage.

\section{$\mathrm{T}$}

$\mathrm{HE}$ effect of changes in pressure upon the ionization of gases has been quite extensively studied at pressures less than an atmosphere, but not so much attention has been paid to the higher values. The first workers in this field studied the change in spark potential difference and a number of such papers ${ }^{1}$ has been published. The results obtained are most contradictory except that the relation between potential difference and gas pressure is a linear one up to 8 or 1o atmospheres. Above that, nothing consistent has been found.

Recently there has appeared a series ${ }^{2}$ of papers on the effect of increased pressure upon ionization due to radium; all showing that, up to at least $\mathrm{I} 7$ atmospheres, the leakage current increases; in some cases the relation is practically linear. W. Wilson ${ }^{3}$ has studied the increase in natural ionization of a closed vessel and also finds this linear relation for pressures greater than 4 atmospheres.

In planning the present work, the intention was to examine the change in disruptive discharge with pressure, but it was found desirable to use instead the point discharge studying the relation between current and potential difference. This particular form of discharge has been carefully investigated by a number of observers, but only Tamm ${ }^{4}$ and Ewers ${ }^{5}$ have studied to any extent the effect of pressure, and that for values less than one atmosphere. Instead

${ }^{1}$ Wolf, Wied. Ann., 37, p. 306, 1889. De Hemptine, Bull. de Belg., p. 603, 1903. Cassinto and Occhiatini, Rend. R. Acc. dei Linc., I5, p. 715, 1906. J. J. Thomson, Cambridge Phil. Trans., 6, p. 326. Ryan, Sibley Journal, I904. C. E. Guye and H. Guye, Phys. Zeitsch., Jan. 15, 1906, p. 62.

${ }^{2}$ Rothé, Comptes Rendus, I47, p. I279, 1908. Erickson, Phys. Rev., 27, p. 473 , 1908. Laby and Kaye, Phil. Mag., 6, I6, p. 216, 1908.

${ }^{3}$ W. Wilson, Phil. Mag., 6, 17, p. 216.

${ }^{4}$ Tamm, Annalen, 6, p. 259, I90I.

${ }^{5}$ Ewers, Annalen, 17, p. 781, 1905. 
of using for electrodes a point and plane, as practically all the workers have done previously, a fine wire and concentric cylinder were employed similar to the apparatus used by Almy. ${ }^{1}$

The luminous effect of the true point discharge is always confined to the point or its immediate neighborhood. This is a region of ionization in which ions of both signs are present. Those of the same sign as the point are repelled, pass out into the non-luminous portion and carry the current from the point to the other electrode, but it is generally assumed that their energy is too small to produce

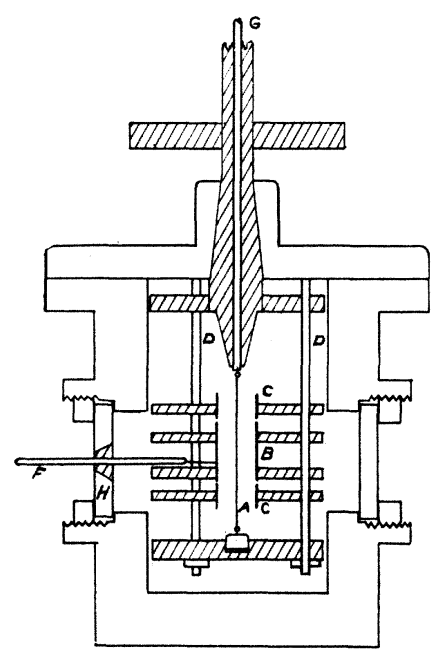

Fig. 1.

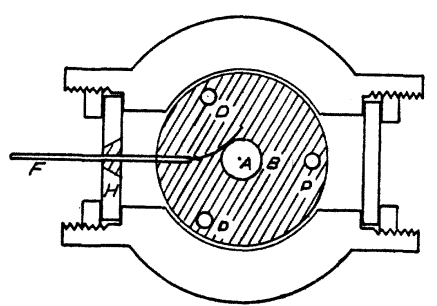

Fig. 2.

ions by collision. In the present work, the wire was covered uniformly with a blue glow when it was charged positively, but when negative in sign the discharge seemed to be localized in spots which were in constant motion. Between these spots could be seen a faint glow which covered more or less of the remainder of the wire.

\section{Apparatus.}

The arrangement of apparatus was comparatively simple. A carefully insulated wire was attached to one terminal of an influence machine whose other terminal was grounded. This wire formed one electrode and was placed in the center of a cylinder which

${ }^{1}$ Almy, Amer. J. Sci., 4, I2, p. I75, I90I. 
[Vol. XXX.

formed the other, and which was connected to earth through a galvanometer that measured the leakage current. The form of the discharge chamber and the arrangement of the electrodes in it are given in Figs. I and 2, which show vertical and horizontal sections.

This chamber was made of brass with walls $1.3 \mathrm{~cm}$. thick; the interior had a depth of $12 \mathrm{~cm}$. and a diameter of $6.7 \mathrm{~cm}$. It was closed with a cast-iron lid which was fastened to it by twelve bolts. Through this lid and carefully insulated from it passed a steel rod $G$ and joined to this rod was the wire $A$ which served as one of the electrodes. The cylindrical electrode $B, 2 \mathrm{~cm}$. long, was held in place by discs of hard rubber which were attached to the lid by the steel rods $D$. In order to obtain an uniform field two guard cylinders $C$ were used. These were connected to the grounded walls of the chamber. ${ }^{1}$ The insulated $\operatorname{rod} F$, tipped with a german silver wire served as a connection between the cylinder $B$ and the galvanometer.

The influence machine was the Toepler-Holtz type, electric-driven and provided with plates of mica three feet in diameter. It was capable of maintaining a difference of potential of approximately 100,000 volts and could furnish a current of at least 600 or 700 microamperes. The potential was regulated by means of a brush discharge occurring between the terminals of the machine and was measured by two Kelvin instruments, a vertical electrostatic voltmeter and an electrostatic balance whose respective ranges were from 1,000 to 16,000 and from 10,000 to 100,000 volts. ${ }^{2}$ The gal-

1 The actual distance between $C$ and $B$ seemed to have very little effect upon the current flowing, provided the gap between them was comparatively small. During the experiment no attempt was made to keep the distance uniform, but it was usually in the neighborhood of $0.02 \mathrm{~cm}$.

${ }^{2}$ No accurate calibration of these instruments was attempted, but, as a check, the spark potential differences between spheres $2 \mathrm{~cm}$. in diameter were determined for varying lengths. The results obtained by the two instruments, when plotted, gave a smooth curve with no discontinuity where the change was made. The results were then compared with those given by Heydweiller (Wied. Ann., 48, p. 220, I893) and were found to be in fair agreement up to 26,000 volts. As far as this reading, the Kelvin instruments gave higher values with a maximum variation of 5 per cent. and an average of 3 per cent. For higher values, much lower results were obtained, but subsequent work made it quite certain that the instrument was more nearly correct than these readings showed, and the lack of agreement was probably due to a different arrangement of apparatus. 
vanometer was of the d'Arsonval type, its maximum sensitiveness being 4.1 $10^{-8}$ amperes per millimeter deflection for the scale distance employed. A shunt box was arranged so that currents up to 800 microamperes could be measured. The gas pressures were determined by a Schaeffer and Budenberg standard gauge, whose range was 100 atmospheres and it was not checked in any way. The instruments were grouped together in such a way that all the variables could be controlled and the readings taken by one observer.

\section{Variations in the Discharge.}

In reading over the literature of the subject, frequent references are made to the discordant results obtained, and how almost imperceptible changes in the conditions would cause most decided variations in the results, especially with the negative discharge. Naturally many such effects were met with in the course of the present work.

Size of Cylinders and Wire. - The first irregularity encountered was that due to the size of the cylinder and wire. With a phosphorbronze wire, number 40 , and a cylinder with an internal diameter of $0.8 \mathrm{~cm}$., the currents obtained for various voltages did not follow any definite law and would not repeat themselves. When a wire of the same material but with a radius of $0.0018 \mathrm{~cm}$. was used, the positive discharge gave concordant results, but the negative one was irregular. When a wire of supposedly the same diameter but made of platinum was introduced, both forms of discharge became regular, i.e., if the values of the current and the corresponding differences of potential were plotted, a smooth curve was obtained. When the cylinder was replaced by another with a diameter of $\mathbf{I} .59$ $\mathrm{cm}$., regular results were obtained even when the radii of the wires were varied from 0.037 to $0.001 \mathrm{~cm} .{ }^{1}$ A possible explanation of these results may be found in the work of $\mathrm{Frank}^{2}$ who obtained

\footnotetext{
1 The size of the wire seemed to have little effect upon the current-potential-difference curves, though, for small currents, the values arranged themselves according to the diameter, the smallest wire giving the greatest current. In passing from a number 40 wire to one having $0.037 \mathrm{~cm}$. for a radius, there was a decided decrease throughout the entire range. Precht (Wied. Ann., 49, p. 150, I893) found that, for small currents, the sharper the point the greater the current flowing, but for large currents all points had about the same effect.

${ }^{2}$ Frank, Wied. Ann., 21, p. 972, I906.
} 
[VOL. XXX.

evidence that ionization by collision was not confined to the immediate neighborhood of the point but occurred at some distance from it. He used a wire and cylinder whose radii were 0.005 and $0.7 \mathrm{~cm}$. respectively and computed that with an electric field of 7,000 volts per centimeter the negative carriers would have suffcient energy to ionize air under atmospheric pressure at a distance of $0.64 \mathrm{~cm}$. from the wire. The positive could do this only at a distance of $0.56 \mathrm{~cm}$., even when the electric force was increased to I0,000 volts per $\mathrm{cm}$. Thus, for the first cylinder employed in this work, the carriers, acted upon by 6,500 volts per $\mathrm{cm}$., would probably reach the cylinder with sufficient energy to ionize the gas and thus change the form of the discharge. As can be seen in the case of the two wires which were supposedly of the same size but probably differed somewhat, it is evident that the dimensions of the apparatus had been unintentionally chosen so that the resulting discharge was on the boundary between a pure point discharge and some other form.

Temperature. - Readings obtained from day to day would rarely agree, and so some data were taken to see if different temperatures have any effect. The air in the room was cooled from $19^{\circ} \mathrm{C}$. down to $13^{\circ} \mathrm{C}$. and then allowed to warm slowly. Observations were made by keeping the machine at a constant voltage, and by taking the readings of the current for the different temperatures. One such series is given.

\begin{tabular}{lccccccc}
\multicolumn{8}{c}{ Positive Discharge. } \\
& $12^{\circ} .3$ & $13^{\circ} .3$ & $14^{\circ} .9$ & $17^{\circ} .4$ & $18^{\circ} .5$ & $19^{\circ} .2 \mathrm{C}$. \\
Temperature, & 6 & 5.83 & 5.75 & 5.86 & 5.75 & $5.75 \mathrm{~cm}$.
\end{tabular}

This would indicate that the effect was very slight, if any, and that the increase in temperature caused a decrease in current.

This effect of temperature has been investigated more or less and the results show a decidedly complicated relation but, in most cases, the effect is small. Warburg ${ }^{1}$ examined the discharge in oxygen and hydrogen at room temperatures and at $175^{\circ} \mathrm{C}$. The presence of ozone complicated the former, but with the latter the current was slightly decreased with increase of temperature for the

${ }^{1}$ Warburg, Drude Ann., 2, p. 295, 1900. 
negative discharge. The positive was affected only to a small extent; with small differences of potential, a decrease; with large differences, an increase, with rise in temperature. Ewers ${ }^{1}$ found that apparently the only effect temperature has is to vary the value of the minimum potential. Thus if the differences between the actual potentials and the minimum potential are considered, temperature has no effect upon the current flowing. He states that the change in minimum potential depends upon a number of factors-gas used, its pressure, etc., and there seems to be no general relation. His range in temperature was from that of the room to that of liquid air. Pringsheim investigated the effect up to $600^{\circ} \mathrm{C}$. and states that a change in temperature from $20^{\circ}$ to $230^{\circ} \mathrm{C}$. caused an increase in the negative discharge of about ro per cent., and the positive discharge showed a similar change, though much smaller. From these results it seems certain that slight changes in the temperature of the gas could not be the cause of any decided variations.

Disruptive Discharge. - It was very early discovered that the potential which could be applied in the case of the positive discharge was limited for high pressures because values of potential were quickly reached which would cause the passage of a spark. On the other hand, no trouble of this kind occurred with the negative discharge. This effect is clearly seen in the following data:

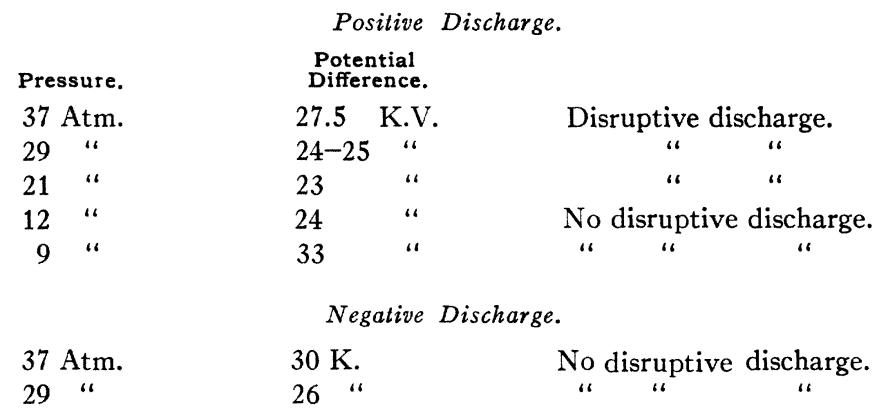

When the positive would stand such high potentials without a spark passing, as it did at 9 and 13 atmospheres, the point discharge was always well established and comparatively large cur-

1 Loc. cit.

2 Pringsheim, Annalen, 24, p. 145, 1907. 
[Vol. XXX.

rents were flowing. The most plausible explanation of this is found in the ease with which the negative ions move. With the wire negative, the electrons would be repelled and, on account of their small inertia, would move rapidly away, leaving a preponderance of positive ions. Thus the decrease in the electric field would be even more abrupt than in the undisturbed state, and so the ionization would be limited to the neighborhood of the wire. This would aid in the establishing of the pure point discharge. In the case of the slow-moving positive ions, the effect would not be so pronounced and conditions similar to the ordinary spark discharge would arise.

Breaking of the Wire.-Considerable trouble was experienced in preventing the wire from breaking. This was due in part to the occasional passage of the disruptive discharge and also to the vibrations which the wire made when the current was passing. The vibrations were very rapid and seemed to be uniformly distributed in all planes. This tended to weaken the wire at its points of attachment. A wire large enough to eliminate these vibrations was tried, but it raised the potential necessary to give the required currents to such high values that it had to be discarded. ${ }^{1}$ Finally platinum wire was used exclusively and was mounted by means of rings as shown in Fig. I. If only moderate currents were allowed to flow, breakages were very infrequent.

Changes in the Surfaces of the Electrodes. - The cylinders were made of brass and, of course, tarnished somewhat after they had been in the discharge chamber for some time. Later they were silvered and then did not tarnish so quickly, but usually showed a mottled appearance. In three different cases, at atmospheric pressure, it was found that the polished cylinders gave values which were slightly lower than those given by the tarnished ones. Individual values could be found that would give the opposite of this, but when comparatively large currents were used and the discharge appeared to be normal, this result was obtained.

\footnotetext{
${ }^{1}$ Although the machine could furnish I00,000 volts, values much above 25,000 could not be used without remodeling the entire arrangement of apparatus because of the great leakage which occurred from all points of the system at these high potentials.
} 
Fig. 3 gives a curve which shows the effect upon the discharge when the wire becomes corroded. In this case number 40 phosphorbronze wire was used and when taken from the container was found to be covered by quite a thick layer of oxide which would obviously roughen the wire. Fig. 4 would indicate that the minimum potential was alone affected.

Source of Electricity. - Since the static machine furnished a constant current, it was thought that possibly the machine did not supply, at all times, sufficient current for the point discharge since the machine had to maintain as well a brush discharge used as a potential regulator. This was tested by placing a high resistance between the terminals of the machine and connecting the electrodes in parallel with a part of this resistance. Then the total current

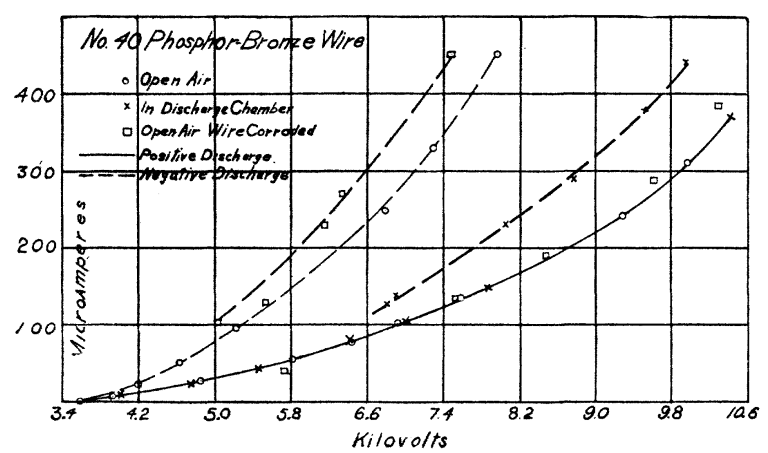

Fig. 3.

furnished by the machine was varied by changing the speed at which it was run. The maximum current used in the discharge chamber during this test was 50 microamperes and the total current furnished was varied from I 50 to 300 microamperes without any effect upon the curves obtained. Consequently it is safe to conclude that the machine was capable of furnishing all the current that the discharge required. Pringsheim ${ }^{1}$ found that the same variations were present whether he used a static machine or a high potential storage battery.

Discharge Chamber. - When the electrodes were introduced into

${ }^{1}$ Loc. cit. 
the discharge chamber, the value of the current for a given difference of potential gradually fell for both forms of discharge. To see if this effect was due to the shielding of the electrodes, they were left in open air but were surrounded by a grounded cylinder of sheet iron, open at both ends. The current was the same as in open air. Similar results were obtained when the discharge took place in the chamber without the disc $H$ (Fig. I) in place. As soon as the chamber was completely closed, the decrease mentioned above appeared. If the disc were removed after the gas had carried the current for some time with the disc in place, values could be obtained which would lie between the two sets just described. The insulation of the rod $F$ was tested and found to be good, so that could not be the reason for this variation.

It has been the experience of a number of experimenters who have worked with electrical discharges where it is necessary to enclose the electrodes, that similar phenomena frequently appear and no very good explanation could be given. Thus Tamm ${ }^{1}$ and Zeleny ${ }^{2}$ found that a glass vessel gave this effect most markedly, whereas vessels of cast iron or brass showed either no effect at all or else it was greatly diminished. Zeleny and $\mathrm{Almy}^{3}$ overcame the difficulty by keeping fresh air circulating through the discharge chamber. Warburg ${ }^{4}$ found that by a careful purifying of the gases these changes could be eliminated.

It was found that this effect could be observed with both positive and negative discharges, though occasionally the former would be unaffected as shown in Figs. 3 and 4. This would tend to indicate the effect of impurities, for ozone was always produced and it has a greater effect upon the negative than upon the positive discharge. Some attempt was made to find the dependence of this decay upon time and, as the effect was more noticeable at high pressures than at low, currents given by a definite potential at 28 atmospheres were observed every minute until a number of readings had been obtained. They did not seem to follow any definite law but gave

${ }^{1}$ Loc. cit.

${ }^{2}$ Zeleny, Phys. Rev., 25, p. 305.

${ }^{3}$ Loc. cit.

${ }^{4}$ Warburg, Drude Ann., 2, p. 295, 1900. 
most irregular results, even occasionally increasing for a reading or two instead of decreasing. They apparently follow to a limited extent the form $i=K e^{-a t}$ but the variations were so great that it could not be said definitely that this law held.

The Gas. - The entire work was with air which was first passed through the purifiers of the liquid air machine and so contained very little carbon dioxide or water vapor, but some oil vapor may have been present. This air was stored under high pressure in a steel cylinder and a large quantity was obtained at one time. Thus, in taking most of the data during August the same air was used

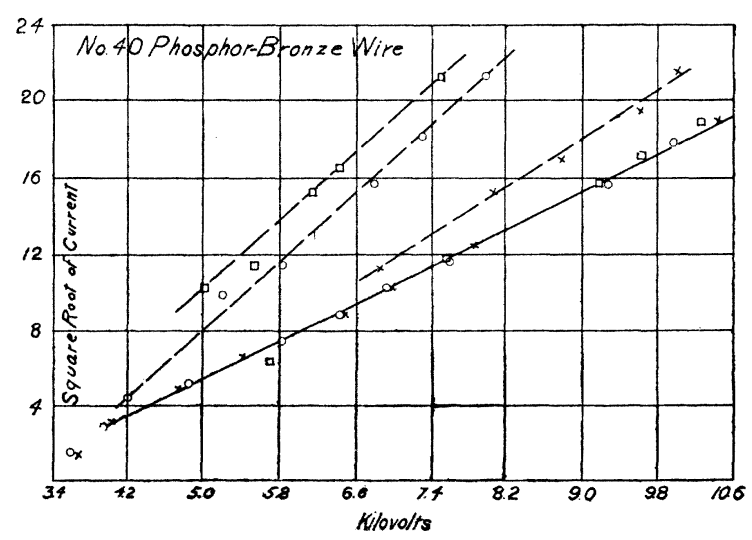

Fig. 4.

throughout, therefore the variations could not be due to the differences in the chemical constituents. A few runs were made with carbon dioxide, but it was found even more unsatisfactory than air.

When the gas was left in the chamber for several hours without a discharge passing, it was found that the current would be less than that obtained from fresh gas, but tended to increase as the discharge was allowed to continue. This could not be due to temperature change, for fresh gas and gas which had been in the container for about fifteen minutes gave identical results. After observing this an attempt was made to get consistent results by leaving the gas in the chamber for a number of hours but without success. 
Possibly the room in which the experiments were carried on may have had something to do with the trouble, for it was in the basement and had no outside windows, though there were large doors on two sides of it which opened into light airy rooms and one of these doors was open constantly. Practically all the data were taken between April and August and most of the observations were made during July and August. It was found that if the discharge took place in the open air during the spring, fairly consistent results were obtained but, when the same thing was tried in the summer, nothing of any value resulted.

Some of these variations may be due to unstable conditions in the discharge itself. For example, Chattock ${ }^{1}$ found that, in hydrogen, the values of the velocities differed among themselves by amounts so large that they could not be attributed to experimental errors. The same thing was noticed in air, but not to so great an extent. Evidently there are a number of factors which contribute to these variations, and this fact makes it difficult to say that a particular result is due to one of these factors and not to another. Thus the lessening of the discharge on July 27 was attributed to the polished surface of the cylinder. This explanation looks plausible; but, on the other hand, no explanation can be given for the sudden increase on August 12. Thus much of the preceding discussion can be considered only as plausible explanations rather than definite effects of specific causes.

\section{REsults.}

The work was almost entirely limited to finding the relation between current and impressed difference of potential, and curves of the form shown in Fig. 3 were always obtained. In Fig. 4 these same data are plotted with differences of potential as abscissæ and square roots of current as ordinates. The points determine more or less definitely a series of straight lines. This result is interesting, for Thomson ${ }^{2}$ has developed, for the particular electrodes used in this work, an equation which would be represented by a similar

\footnotetext{
1 Thomson, Conduction of Electricity through Gases, 2d ed., p. 504.

${ }^{2}$ Chattock, Phil. Mag., V., 48, p. 40I, I899. Chattock, Walker and Dixon, Phil. Mag., VI., I, p. 79, I90I.
} 
curve. It has been found that, at atmospheric pressure, this relation between current and difference of potential holds in almost every case. The exceptions are-for the negative discharge when out of the container, though the data in Fig. 4 follow this equation; also when a small static machine was used, in which case irregular results were obtained for the small currents used. As a result of the almost universal agreement, it is safe to say that Thomson's equation represents the general relation between current and potential difference when the current is larger than ro microamperes, so in plotting the curves the square roots of the currents were always used as ordinates.

As the results for small currents did not fit the equation, the various approximations made in its development were investigated to see if they would have any effect. This departure is noticeable for currents of several microamperes and the terms neglected when such currents were used would introduce an error of less than I per cent.; hence their omission cannot explain the variation.

Thomson's complete equation is of the form

$$
\begin{aligned}
i & =\frac{K}{2 b^{2}}\left(V-V_{0}-\alpha\right)^{2} . \\
k & =\text { the velocity of the ions under unit electric force. } \\
a & =\text { radius of the wire. } \\
b & =\text { radius of the cylinder. } \\
V_{0} & =\text { minimum potential difference for disruptive discharge. } \\
\alpha & =\frac{1}{2} \sqrt{C} \log \frac{2 C}{\frac{i}{K}} a^{2}
\end{aligned}
$$

$C$ is a constant of integration and cannot be larger than $i / K a^{2}$.

By assuming the critical spark length as $0.001 \mathrm{~cm}$. and the maximum intensity as 100 electrostatic units, which is really less than its true value, the numerical values of the constants can be computed for the apparatus used. The two forms of discharge give the following:

$$
\begin{aligned}
& i^{\frac{1}{3}}=17.3(V-\mathrm{I} .43) \text { positive. } \\
& i^{\frac{1}{1}}=20.5(V-\mathrm{I} .45) \text { negative. }
\end{aligned}
$$


$\alpha$ is a function of the current and thus varies considerably. In the positive discharge, for 0.1 microampere it is equal to $\mathrm{I} .2 \mathrm{I}$; for 400 microamperes, it is 1.65 . In the above equations, the average values are used. The experimental values of these constants were determined for three sets of curves obtained with the same sized wire and cylinder and are as follows:

$$
\begin{aligned}
& i^{\frac{1}{1}}=38.7(V-8.72) \text { positive. } \\
& i^{\frac{1}{2}}=45.7(V-8.32) \text { negative. } \\
& i^{\frac{1}{2}}=45.2(V-8.28) \text { negative. }
\end{aligned}
$$

The agreement between the two sets is not good and the discrepancies are too great to be attributed to experimental errors. Of course the electric intensity used is lower than its true value, so possibly the agreement for $\alpha$ should be better than is indicated; but no error of that kind can account for the lack of agreement in the case of the other constant. This divergence may de due to lack of symmetry in the apparatus, so that the discharge might be concentrated along certain radii or radial planes instead of being uniformly distributed. The equation is also developed on the assumption of only one kind of ions being present in the non-luminous portion, while Wilson ${ }^{1}$ and Campbell ${ }^{2}$ in their work on the mass of these ions have shown that ions of both signs may be present.

The work of Frank ${ }^{3}$ offers the best explanation for the variation in the slopes of the two sets of curves. He found that if the velocity of the ion due to unit electric intensity is determined in the neighborhood of the small electrode, the values varied most decidedly among themselves and were much larger than those determined by other workers. On the other hand, when he measured this quantity at some distance from the point, he obtained results in close agreement. This quantity is assumed a constant in Thomson's equation.

Figs. 5, 6, 7 and 8 show the effect upon the point discharge of an increase in gas pressure. The values plotted in Fig. 5 are the average of a number of runs with the wire charged negatively.

${ }^{1}$ C. T. R. Wilson, Phil. Trans., A, Vol. CXCII., p. 403, 1899.

${ }^{2}$ Campbell, Phil. Mag., 6, 6, p. 618, 1903.

${ }^{3}$ Loc. cit. 
As was to be expected, the increase in pressure caused a great decrease in current, which might in part be attributed to the decrease

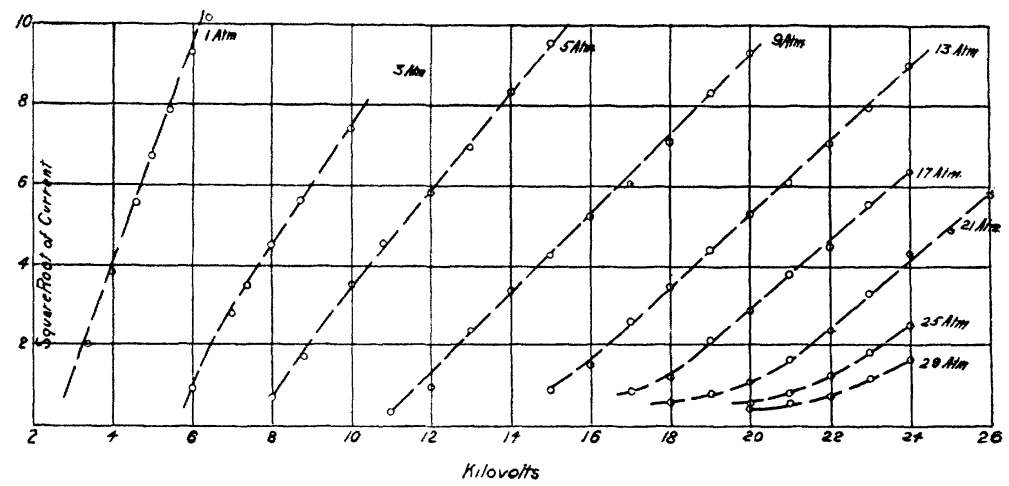

Fig. 5.

in velocity of the ions as shown by the change in the slope of the curves, and also to the much higher value of the minimum potential.

In Fig. 6 are plotted the values obtained with a phosphor-bronze wire using currents up to 400 microamperes. The irregularities

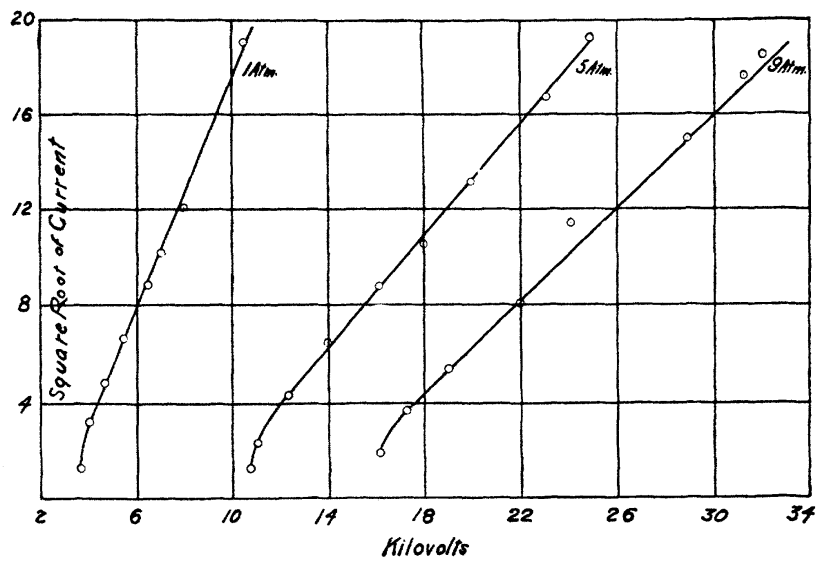

Fig. 6.

seen in the last two points on the curve at 9 atmospheres are probably due to inaccuracies in the voltmeter readings. These curves show very noticeably a variation from the straight line for values less 
[Vol. XXX.

than Io microamperes ${ }^{1}$ and this same variation can be seen in curve 7 which indicates the effect of pressure upon both forms of discharge. $^{2}$

It has been found that these small currents satisfy much better an equation suggested by Warburg of the form

$$
i=A V(V-M) \text {. }
$$

$A$ is a constant and $M$ represents the minimum potential. Most observers have found that this equation seems to be followed quite generally when the point and plane are used as the electrodes.

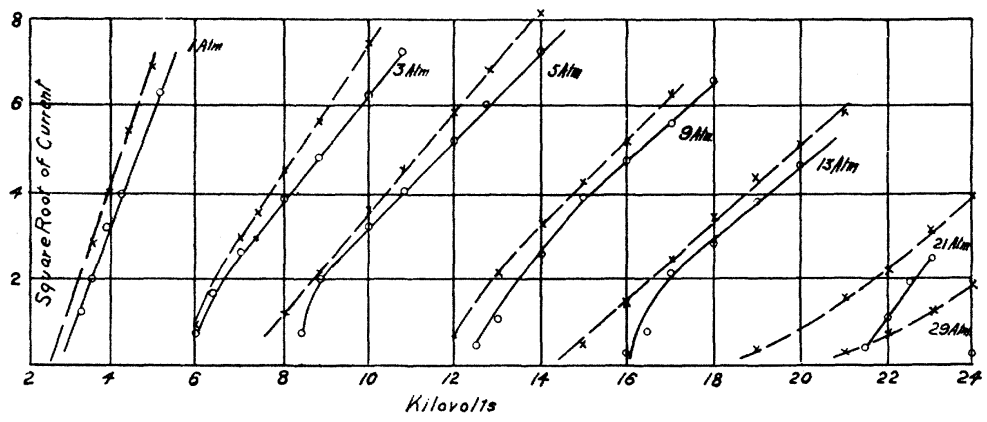

Fig. 7.

No explanation can be given why in this work, the change from one equation to the other should appear for the small currents.

At a pressure of 17 atmospheres or more, the sign of this curvature reverses and the curves approach the $X$-axis more slowly than they should. This can be seen in Fig. 7 , but is especially noticeable in Fig. 8. The data for this last set of curves were taken on August 12 and no reason is known for the unusual departure from the normal form. The general form of these curves is similar to those obtained when some agent is used to ionize a gas and the electric field between the electrodes is increased to such a strength that ionization by collision occurs. Hence it is natural to suppose that

${ }^{1}$ Curves of the same form have been obtained when the data published by other observers have been plotted with the square root of current as ordinates. Especially good examples were in the work of Tamm (loc. cit.), Zeleny (loc. cit.) and Sieveking (Ann., I, p. 299, 1900) all of whom used the point and plane electrodes.

${ }^{2}$ In all the subsequent curves, the negative discharge will be represented by dotted lines and the positive, by full lines. 
some other ionizing agent was present, which furnishes ions for these small currents and that the electric field had a very small part in producing them. This view is strengthened by the fact that pressures of 60 or 70 atmospheres could not entirely stop the negative current produced by a potential difference of 25,000 volts. The ions producing the current could hardly have been formed by collision, and the fact that it remained practically constant throughout wide ranges of pressures would indicate a saturation current.

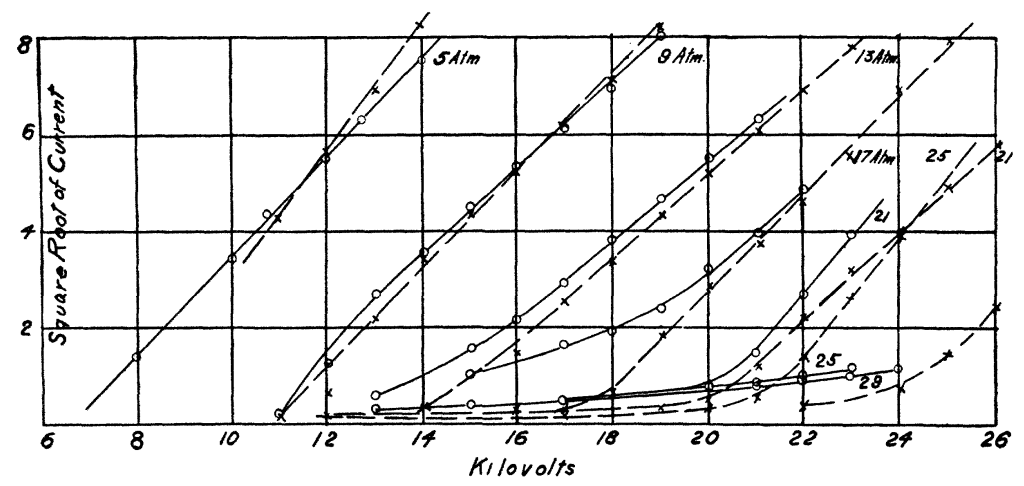

Fig. 8.

The work of Rothe and others mentioned in the early part of this paper would indicate that this effect should be more noticeable at high pressures than at low ones.

As a result of these curvatures, the minimum potential, which is characteristic of the point discharge, was not sharply defined. Other observers have usually found that the discharge began at a certain potential and then stopped at a lower one. They also mentioned that the current did not die away gradually but fell abruptly to zero. Most of them were working with galvanometers about roo times as sensitive as the one used in this work; and the big influence machine did not work satisfactorily when run slowly enough to give the low potentials needed. Consequently none of these effects were noticed. At the high gas pressures it looks as though the minimum potential depends upon the sensitiveness of the galvanometer used, and if the true point discharge is concealed by some other discharge, then the minimum potential can have but little significance. 
[VoL. XXX.

According to Thomson's equation, the ratio of the slopes of the curves for the different discharges should be the square root of the velocities of the two kinds of ions. Trying this for the constants in the equations given on page 732 , they give as the ratio 1.37, whereas Chattock found it to be 1.36 . This close agreement is a matter of chance, for other curves give values varying from 1.22 to I.48. Though two cylinders of unequal size were used, it was impossible to see if the slopes of the lines varied inversely as the radii because the results with the small cylinder were so irregular.

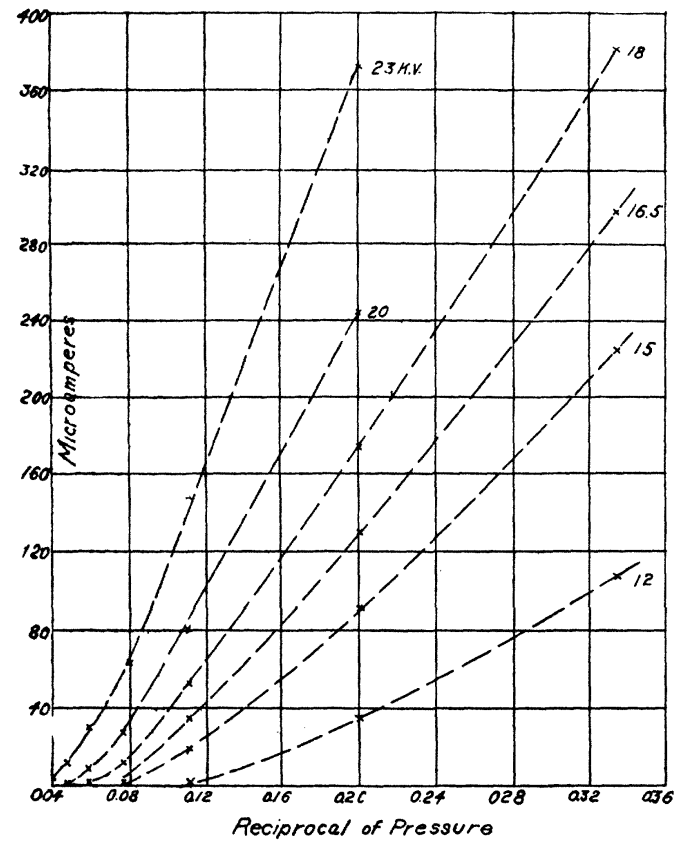

Fig. 9.

If the assumption is made that the velocity of the ions is inversely proportional to the gas pressure, then certain relations may be deduced from the equation. Thus if the voltage is kept constant, then the current must vary inversely with the pressure. This holds for large currents and rather high voltages, as shown in Fig. 9. To obtain this curve, the lines in Fig. 5 had to be exterpolated, but no values greater than 400 microamperes were used, and Fig. 6 
shows that the point discharge still holds for that current. In a similar way the equation says that, for constant current, the voltage must increase as the square root of the pressure and Figs. Io and I I

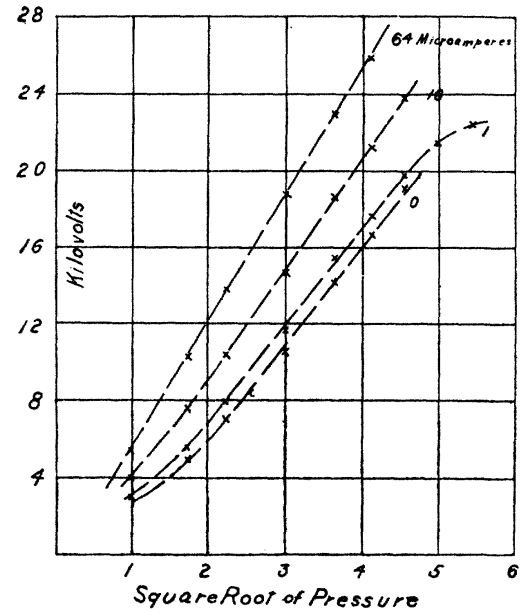

Fig. 10.

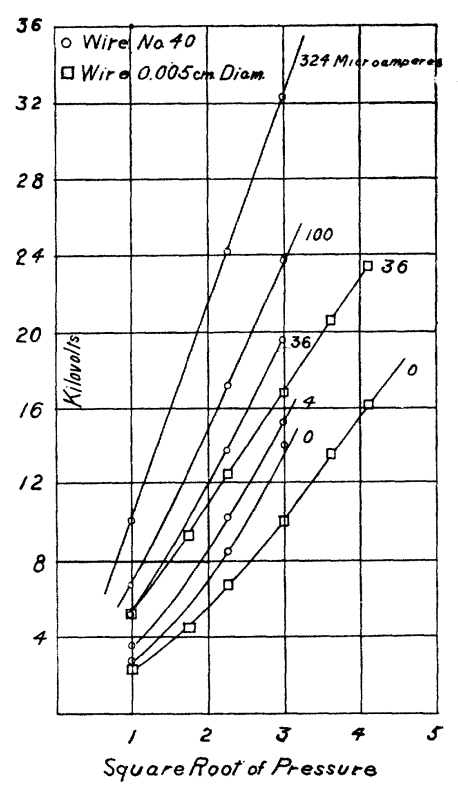

Fig. 11.

show that this condition is satisfied for large currents. Fig. I I also indicates that the size of the wire has a very decided effect upon these curves. These figures tend to confirm the idea that the velocity of the ions vary inversely as the pressure.

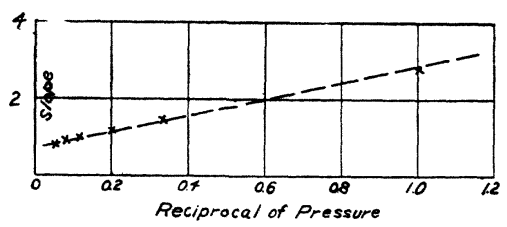

Fig. 12.

If the slopes of the lines given in Fig. 5 are plotted as ordinates and the reciprocals of pressure as abscissæ, a straight line results as shown in Fig. I2. According to Thomson's equation, this would mean that the velocity varies inversely as the square of the pressure, 
[Vol. XXX.

which is antagonistic to the results given in Figs. 9, Io and I I, but it is in approximate agreement with Tamm's ${ }^{1}$ work, for he found that the following equation held for pressures less than one atmosphere:

$$
i_{x}=i_{76}\left\{\frac{76}{x}-\sqrt[3]{\frac{v}{\mathrm{IO}^{5}}} \log \frac{76}{x}\right\}^{2}
$$

\section{Conclusions.}

I. The relation between current and impressed potential difference follows the equation

$$
i^{\frac{1}{2}}=A(V-M)
$$

for currents varying from 10 to 400 microamperes. For currents less than ro microamperes, the results are irregular.

2. Pressure has a very great influence upon the true point discharge, due both to an increase in minimum potential and to a decrease in the velocity of the ions. The curves would indicate that the velocities of the positive and the negative ions become more nearly equal at high pressures, due probably to the loading down of the electron by neutral molecules.

3. It was found that the slopes of these straight lines vary inversely as the gas pressure; that, for a given current, the corresponding voltages vary as the square root of the pressures; that the current given by a constant difference of potential vary inversely as the pressure. These last two relations support the view that the velocity of the ions decrease directly as the pressure, while the first one indicates that the velocity varies inversely as the square of the pressure. From the present work there is no means of telling which is the correct relation.

4. These relationships were found to hold up to 17 atmospheres, but above that currents large enough could not be obtained by the present arrangement of apparatus to test them.

5. At the high pressures, phenomena were observed which might be explained by the presence of an ionizing agent, other than the electric field, which tended to mask the true point discharge.

${ }^{1}$ Loc. cit. 
The experimental work was done in the Physical Laboratory of Cornell University during the spring and summer of 1907. The author wishes to express his appreciation of the advice and suggestions offered by the various members of the department, especially Professors Merritt and Shearer.

UNIVERSITY OF WISCONSIN,

MADISON, Wis. 